\title{
Atomization of oxygen nanodispersions in isotonic saline for applications in aerosol therapy.
}

\author{
$\underline{\text { Marcin Odziomek }}^{\mathbf{1}}$, Katarzyna Dobrowolska ${ }^{1}$, Karol Ulatowski $^{\mathbf{1}}$, Pawel Sobieszuk ${ }^{\mathbf{1}}$, \\ Tomasz R. Sosnowski ${ }^{1}$
}

1. Faculty of Chemical and Process Engineering, Warsaw University of Technology, POLAND, Warsaw, Waryńskiego 1 Street, 00-645 Warsaw, E-mail: marcin.odziomek@pw.edu.pl

\begin{abstract}
We tested the possibility of using dispersions of oxygen nanobubbles as new carriers for inhalation drugs delivered from medical nebulizers. Their successful application may become attractive in the treatment of lung diseases, giving the possibility of simultaneous delivery of the drug and improve the oxygen supply. The results show that after atomization nanobubbles are preserved in aerosol droplets. Simultaneously, the aerosol size distribution and output rate do not change.
\end{abstract}

Keywords - nanobubbles, aerosol therapy, oxygen therapy, inhalation, nebulization, nebulizer, droplet size distribution, volume median diameter.

\section{Introduction}

Nebulization is the aerosolization method of liquid drugs delivered to the respiratory system via inhalation. It is preferentially used for patients in severe health conditions (e.g., patients under mechanical ventilation support) and often accompanies oxygen therapy [1]. The high efficiency of inhalable treatment demands micrometer-sized aerosol produced from solutions or suspensions of the active pharmaceutical active ingredient (API) in special medical devices called nebulizers. According to a mode of action: jet nebulizers, vibrating mesh nebulizers, and ultrasonic nebulizers can be distinguished.

In this work, we tested the possibility of using dispersions of oxygen nanobubbles (DONs) as an alternative liquid carrier for APIs aerosolized in different kinds of nebulizers. Nanobubbles are nanoscale gas spheres suspended in liquids with numerous characteristics distinguishing them from their macroscale counterparts [2, 3]. Excellent stability, high internal pressure, the enormous surface-to-volume ratio, and the high gas dissolution rate are essential features of NBs, leading to many promising applications in various fields of advanced science and technology [4, 5]. As stated, the nanobubbles dispersions influence the proliferation of cells and even whole-organism growth $[6,7]$. Additionally, they can assist in treating numerous diseases, including local hypoxia, e.g., ischemic foot $[8,9]$. The successful application of DONs in the role of the carrier in aerosol therapy would have potentially an additional advantage of introducing much more oxygen - along with the aerosolized drug - to the surface of the respiratory system. This may improve the oxygen supply, especially in the respiratory diseases associated with hypoxia.

\section{Methodology}

The generation of nanobubbles was performed using the membrane method and setup with flat membrane and mechanical stirrer (originally designed by our research team). Generation processes were carried out in $0.9 \% \mathrm{NaCl}$ (physiological saline, B.Braun, Germany). The dispersions were atomized using four medical nebulizers with a different mode of action: jet nebulizer (Pari Boy SX, PARI Pharma GmbH, Germany), two vibrating mesh nebulizers - VMN (Aerogen Solo, Aerogen, USA and Intec Mesh Twister, Intec Medical, Poland) and ultrasonic nebulizer (Thomex MBU, Medbryt, Poland). 
The output rate of aerosol released from nebulizers was measured gravimetrically, and the volumetric droplet size distribution was measured using a laser diffractometer (Spraytec, Malvern Panalytical, UK). The emitted aerosols were also collected in a glass vessel that allowed to convert them to liquid again. Dispersions at each stage were analyzed using Dynamic Light Scattering (Zetasizer NanoZS, Malvern Panalytical, UK) for number-averaged densities of the size distribution of nanobubbles.

\section{Results}

The results show that after atomization, nanobubbles are still present in the liquid collected from condensing aerosol. Simultaneously, there are noticeable differences in size distributions of oxygen nanobubbles present in dispersions based on the isotonic solution before and after atomization with different nebulizers. It can be seen that their average size decreases in the case of ultrasonic and jet nebulizers and increases in both devices with vibrating mesh. (Fig. 1).

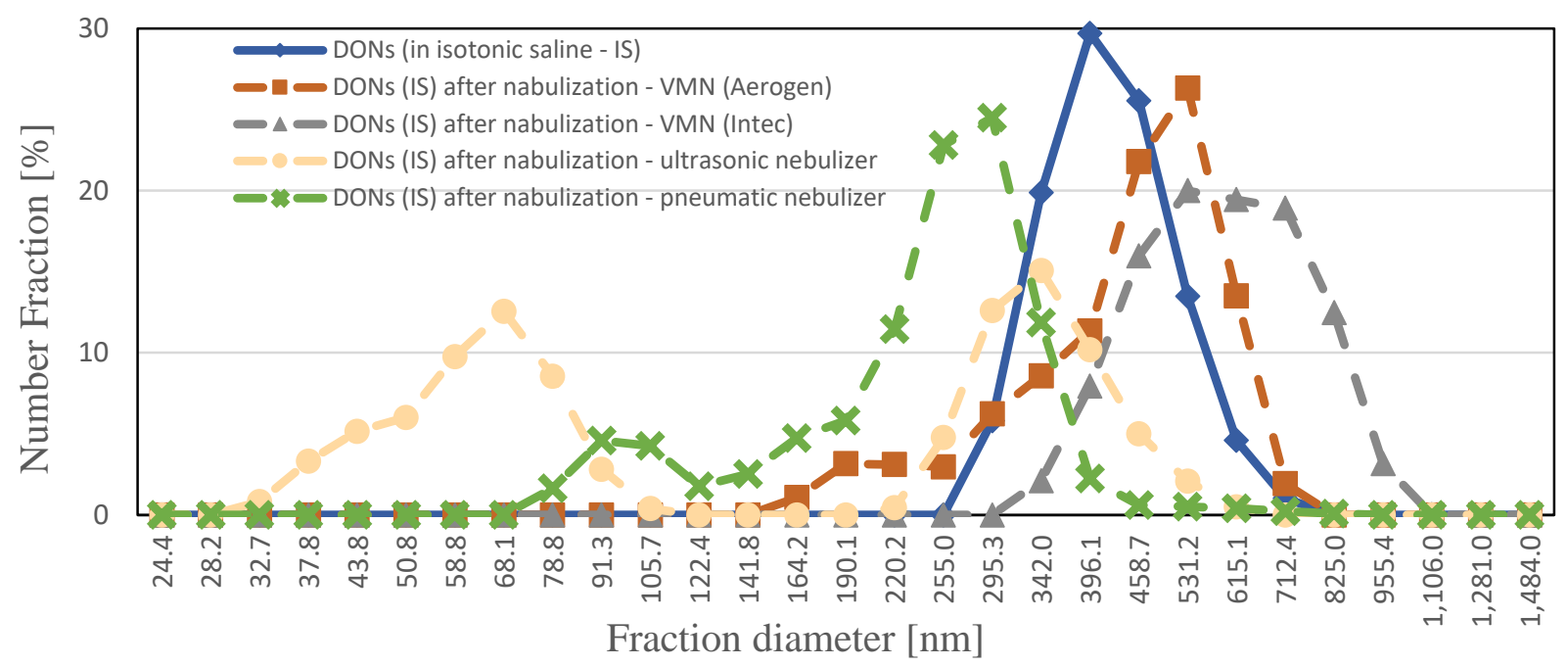

Fig. 1. The size distribution of nanoobjects in dispersions based on isotonic saline before and after atomization in different nebulizers.

The comparison of aerosol generated from liquids with and without (w/out) nanobubbles shows that the size of droplets and output rate are similar (Fig. 2, Fig. 3).

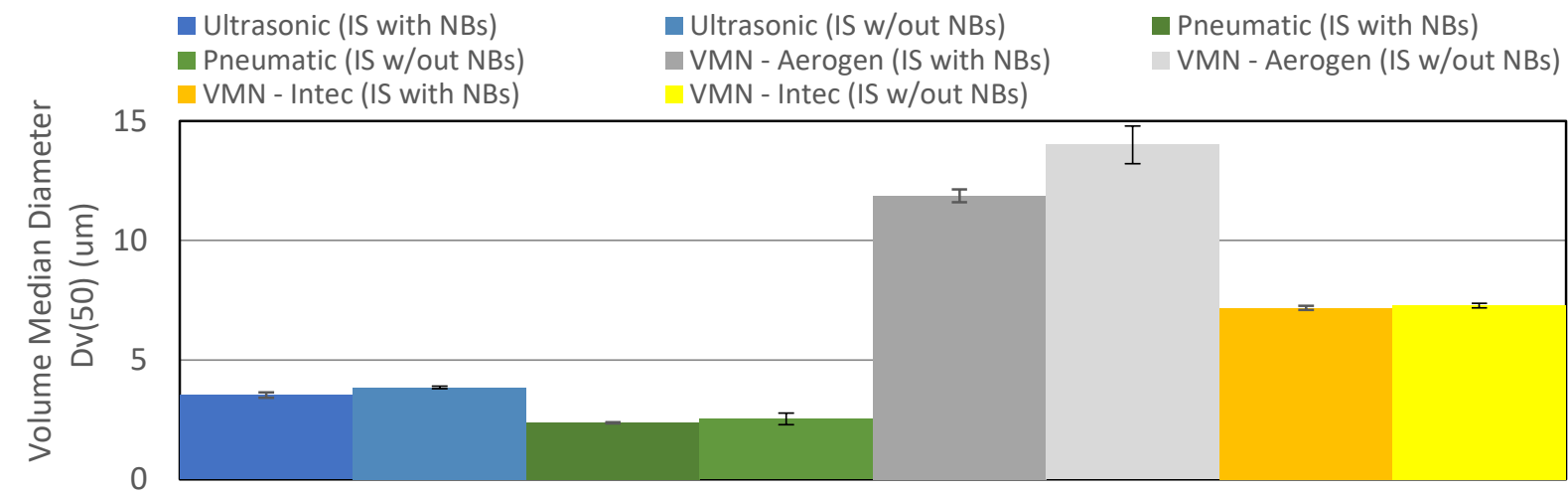

Fig. 2. Volume median diameter of aerosols dispersed by different medical nebulizers from isotonic saline and DONs in isotonic saline. 


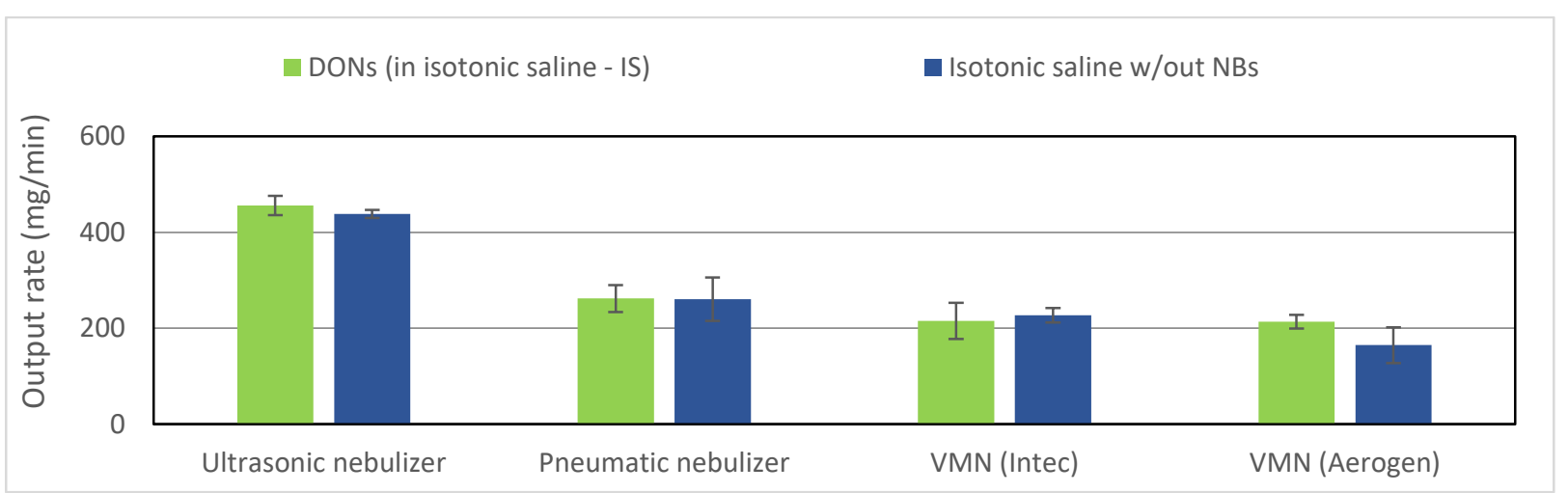

Fig. 3. Output rate of aerosols dispersed by different medical nebulizers from isotonic saline and DONs in isotonic saline.

\section{Conclusion}

The addition of nanobubbles to a nebulized solution does not require changing the construction of commercially available nebulizers or other parameters of the atomization process to obtain fine droplets with a huge potential in aerosol therapy. Simultaneously, initial results indicate that the aerosol generation process in medicinal nebulizers does not change the stability of DONs.

\section{Acknowledgments}

Work supported/Studies were funded by BIOTECHMED-1 project granted by Warsaw University of Technology under the program Excellence Initiative: Research University (ID-UB).

\section{References}

[1] Dhanani, J., Fraser, J. F., Chan, H. K., Rello, J., Cohen, J., Roberts, J. A. (2016). Fundamentals of aerosol therapy in critical care. Critical care (London, England), 20(1), 269. https://doi.org/10.1186/s13054-016-1448-5

[2] Azevedo, A., Oliveira, H., Rubio, J. (2019) Bulk nanobubbles in the mineral and environmental areas: Updating research and applications. Adv Colloid Interface Sci 271:101992. doi: 10.1016/j.cis.2019.101992

[3] Alheshibri, M., Qian, J., Jehannin, M., Craig, V.S.J. (2016) A History of Nanobubbles. Langmuir 32:11086-11100. doi: 10.1021/acs.langmuir.6b02489

[4] Ulatowski, K., Sobieszuk, P., Mróz, A., Ciach, T. (2019) Stability of nanobubbles generated in water using porous membrane system. Chem Eng Process - Process Intensif 136:62-71. doi: 10.1016/j.cep.2018.12.010

[5] Wang, Q., Zhao, H., Qi, N., et al (2019) Generation and Stability of Size-Adjustable Bulk Nanobubbles Based on Periodic Pressure Change. Sci Rep 9:1-9. doi: 10.1038/s41598-01838066-5

[6] Park, J.S., Kurata, K. (2009) Application of microbubbles to hydroponics solution promotes lettuce growth. Horttechnology 19:212-215

[7] Ebina, K., Shi, K., Hirao, M., et al (2013) Oxygen and Air Nanobubble Water Solution Promote the Growth of Plants, Fishes, and Mice. PLoS One 8:2-8. doi: 10.1371/journal.pone.0065339

[8] Sayadi, L.R., Banyard, D.A., Ziegler, M.E., et al (2018) Topical oxygen therapy \& micro / nanobubbles : a new modality for tissue oxygen delivery. 363-374. doi: 10.1111/iwj.12873

[9] Wang, S., Yin, C., Han, X., et al (2019) Improved Healing of Diabetic Foot Ulcer upon Oxygenation Therapeutics through Oxygen-Loading Nanoperfluorocarbon Triggered by Radial Extracorporeal Shock Wave. 2019: 